\title{
Student Objections to and Understanding of Non-Cartesian Unit Vector Notation in Upper-Level E\&M
}

\author{
Brant E. Hinrichs \\ Department of Physics, Drury University, 729 N. Drury Lane, Springfield, MO 65802
}

\begin{abstract}
The upper level E\&M course (i.e. based on Griffiths [1]) involves the extensive integration of vector calculus concepts and notation with abstract physics concepts like field and potential. We hope that students take what they have learned in their math classes and apply it to help represent and make sense of the physics. Previous work showed that physics majors at different levels (pre-E\&M course, post-E\&M course, 1st year graduate students) had great difficulty using non-Cartesian unit vector symbols appropriately in a particular context [2]. Since then we have developed a series of problems they work on in groups and discuss as a whole class [3] to help them confront and resolve some of their difficulties. This paper presents those problems, typical in-class group responses, and three years of post-test data. Results show that students have (i) a very strong initial negative reaction to the vagueness of the $\hat{r}$ symbol, and (ii) an improved functional understanding of the notation as demonstrated by a better ability to use the symbols appropriately.
\end{abstract}

Keywords: spherical unit vectors, cylindrical unit vectors, position vector, intermediate electrodynamics, board meeting PACS: $01.40 . \mathrm{Fk}$

\section{INTRODUCTION}

This paper presents initial results from a study designed to help students better use and understand spherical and cylindrical unit vectors in the upper-level E\&M course [1]. Previous work showed that just 2\% of students at many different levels and types of institutions were able to write appropriate 3-d position vectors using non-Cartesian unit vector symbols [2]. Understanding $\bar{r}$, the 3 -d position vector, is important because of its ubiquity in the upper-level E\&M course [2]. Since that 2010 paper, no other work on the problem has been reported in the literature.

In addition to collecting paper and pencil data for the 2010 paper, we also conducted videotaped semistructured interviews with twelve of the students from that population. Analysis of transcripts from the

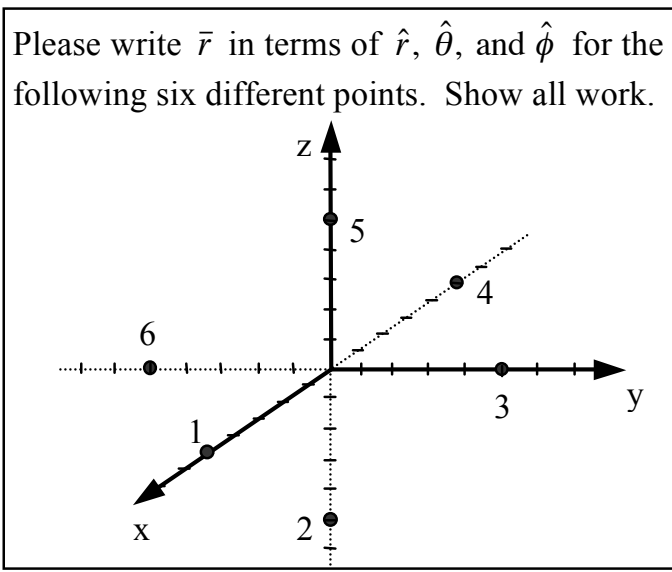

FIGURE 1. Pre-test from [2] given as homework prior to inclass work. (Expected answer: $\bar{r}=5 \hat{r}$ for all six points) twelve showed that (i) most had good working knowledge of Cartesian unit vectors and spherical coordinates, (ii) at least half could correctly draw spherical unit vector triads of $\hat{r}, \hat{\theta}$, and $\hat{\phi}$ at different points in space, and (iii) none changed their minds but strongly defended their original responses even when shown correct answers.

These results, especially (ii), suggested that a major student difficulty was making sense of the unit vector symbols themselves. So a series of problems was developed to help lead students from what they did understand to what they did not. The rest of this paper presents the context for this study, the problems themselves, typical group and class responses, and three years of individual post-test data. Along the way we discuss some possible implications for teaching as well as some ideas for future research.

\section{CONTEXT}

The data in this paper are from the upper-level E\&M course at Drury University. It was taught using a University Modeling (UM) approach [4] that centered around Modeling Discourse Management [5], a learning-community approach designed to help students understand that science is tentative and evolving and that knowledge and meaning are constructed and shared through dialogue. One important feature of UM is the explicit emphasis on the coordination of multiple representations. The problems presented in this paper are based on that idea because students who are comfortable using a range of representations in problem solving better approximate 
physics experts, who routinely create and coordinate many different representations (e.g. mathematical, graphical, diagrammatic) in the analysis of a single problem $[6,7]$. All students were $4^{\text {th }}$ or $5^{\text {th }}$ year physics majors. The instructor was the researcher.

The upper-level classes at Drury are small, not always taught every year, and sometimes taught by different faculty, so our data set is quite modest, coming from just the 2011 ( 8 male, 0 female), 2014 $(12 \mathrm{~m}, 2 \mathrm{f})$, and $2017(3 \mathrm{~m}, 1 \mathrm{f})$ classes. We look forward to hearing if researchers working with other student populations find similar results.

\section{CURRICULUM SEQUENCE}

Here we describe the work students were assigned to try to help them develop a functional understanding of spherical unit vector notation.

\section{Homework}

Prior to any in-class work on spherical unit vectors, students are given a homework assignment to read about spherical unit vectors in their text, summarize what they learned, write down questions they have, and complete a conceptual problem (Fig. 1, see [2]) as a pre-test for the days' in-class work.

\section{In-Class Problem \#1}

Once in class, students work in small groups of 2-3 to compare their answers on the pre-test, create solutions on a small (2'x $\left.3^{\prime}\right)$ whiteboard to the following statements:

(a) Plot $\hat{x}, \hat{y}$, and $\hat{z}$ at the following three points:

$$
\begin{aligned}
& r=1, \theta=180^{\circ}, \phi=0^{\circ} \\
& r=1, \theta=90^{\circ}, \phi=0^{\circ} \\
& r=1, \theta=90^{\circ}, \phi=180^{\circ}
\end{aligned}
$$

(b) Plot $\hat{r}, \hat{\theta}$, and $\hat{\phi}$ at the same three points on a different coordinate system.

and "circle up" to find a consensus through a studentlead, whole-class discussion (i.e. board meeting [3]). Part (a) revisits points 1-3 from the pre-test (Fig. 1), and is designed to remind students about using Cartesian unit vectors and spherical coordinates and to introduce the idea that unit vectors don't necessarily have to be anchored at the origin. Part (b) is designed

TABLE 1. Group data from Problem \#1 done in class

\begin{tabular}{c|c|c}
\hline $\begin{array}{c}\text { Problem } \\
\text { Statement }\end{array}$ & $\begin{array}{c}\% \text { of groups that brought } \\
\text { a correct answer to the } \\
\text { board meeting }\end{array}$ & Consensus \\
\hline (a) & $70 \%$ & quick, easy, correct \\
(b) $)^{1}$ & $20 \%$ & challenging, sometimes incorrect \\
\hline
\end{tabular}

${ }^{1}$ Only for 2011, 2017. Was not asked in 2014. to probe their conceptual understanding of the graphical nature of spherical unit vectors. The two parts together take about 60 minutes of class time.

\section{In-Class Problem \#2}

Whether it is correct or not, the consensus from Problem \#1 is set aside and the small groups create solutions on different whiteboards to these statements:

(c) Write a position vector for each of the previous three points in terms of $\hat{x}, \hat{y}$, and $\hat{z}$,

(d) Use the formulas in your book (or back cover of Griffiths) to calculate $\hat{r}, \hat{\theta}$, and $\hat{\phi}$ at the same three points and reconcile with (b),

(e) What do you notice?

(f) Write a position vector for each of the previous three points in terms of $\hat{r}, \hat{\theta}$, and $\hat{\phi}$.

and hold another board meeting to find a new consensus. Part (d) is designed to help students start connecting the graphical, mathematical, and symbolic meaning of spherical unit vectors. Answers to part (d) also allow them to check the previous consensus answer to part (b). Part (e) is designed to help them start making explicit connections between Cartesian and spherical unit vectors, which should now help them do better on part (f) (which was the pre-test). These questions, especially part (f), are more challenging and can take up to 90 minutes to whiteboard and resolve.

After this in-class work, for homework the following week, students read the section in their text about cylindrical unit vectors and wrote about it, but $\hat{s}, \hat{\phi}, \hat{z}$ were never discussed extensively in class.

\section{STUDENT DATA}

All students got the entire pre-test incorrect (as consistent with [2]). For the rest of this section we just report group answers (based on photos of student whiteboards) because those are what drive our small and large-group class discussions. Table 1 shows group data from Problem \#1. Common errors on (a) were drawing all triads at the origin rather than at the points in question, and on (b) were drawing curved arrows showing the actions of $\theta$ (rotating the position vector $\bar{r}$ down from the z-axis) and $\phi$ (rotating $\bar{r}$ clockwise about the $z$-axis) but labeling them as unit vectors, thinking that $\hat{r}$ always points like $\hat{z}$, and mapping $\hat{r}, \hat{\theta}, \hat{\phi}$ directly onto $\hat{x}, \hat{y}, \hat{z}$. Table 2 shows group results from Problem \#2. Errors on (d) were switching labels on the angular unit vectors for point 2 and difficulties with point 3 , either with a sign error for $\hat{r}$ or having incorrect directions for $\hat{\theta}, \hat{\phi}$. 
TABLE 2. Group data from Problem \#2 done in class.

\begin{tabular}{c|c|c}
\hline $\begin{array}{c}\text { Problem } \\
\text { Statement }\end{array}$ & $\begin{array}{c}\text { \% of groups that brought a } \\
\text { correct answer to board meeting }\end{array}$ & Consensus \\
\hline (c) & $100 \%$ & quick, easy, correct \\
\hline (d) & $\begin{array}{c}\text { Calculations }-90 \% \\
\text { Plots }-70 \%\end{array}$ & $\begin{array}{c}\text { took time to resolve issues, } \\
\text { correct }\end{array}$ \\
(f) & 1 group in each class ${ }^{2}$ & $\begin{array}{c}\text { extremely difficult, } \\
\text { instructor heavily involved }\end{array}$ \\
\hline 22011 and 2014 "seeded" this by the instructor [8].
\end{tabular}

The big challenge comes with part (f). Only one group in each class came to the large group with the correct answer and in two of those cases the instructor gave them strong hints ("seeds") [8] to help them get over their initial impasse. In both cases, after seeding, they were able to work out the correct solution on their whiteboard and present it to the rest of the class. But in all three years, having only one group with the correct approach was not enough to convince all the others. The board meetings got into a sharp dispute that could not be resolved without extensive intervention from the instructor. An implication for teaching and future research is to see if consensus is possible to achieve when at least two groups come into the board meeting with correct answers rather than just one.

\section{INTERVENTION AND RESPONSE}

In this section we briefly outline the chain of reasoning used by the instructor to guide the class to coordinate their Cartesian and non-Cartesian representations for point 2 and reach consensus. We then report on the students' response to the conclusion of the argument.

Here is the reasoning. At the end of the day, to be consistent, both representations should give the same answer of $-\hat{z}$. To start, it must be that $\bar{r}_{2}=-5 \hat{z}$ in Cartesian unit vectors. It must also be the case that, in spherical unit vectors, based on answers to part (d), $\hat{r}=-\hat{z}, \hat{\theta}=-\hat{x}$, and $\hat{\phi}=\hat{y}$. So, to be consistent, it is sufficient that $\bar{r}_{2}=5(-\hat{z})=5 \hat{r}$ in spherical unit vectors. No $\hat{\theta}(-\hat{x})$ or $\hat{\phi}(\hat{y})$ contributions are needed. So all three points are written exactly the same, as $5 \hat{r}$. Done!

Students can follow this logic, but the conclusion is very unsettling to them (quotes are from transcripts of audio recordings of the board meetings):

\section{S1: "well then, what? It is completely insane, and stupid. I don't understand it. It doesn't make sense. I don't know why someone invited this, but it doesn't make a bit of damned sense."}

That's the strongest reaction I've observed, and the student was half-joking, but most students find the notation ambiguous and unhelpful and spontaneously say so:

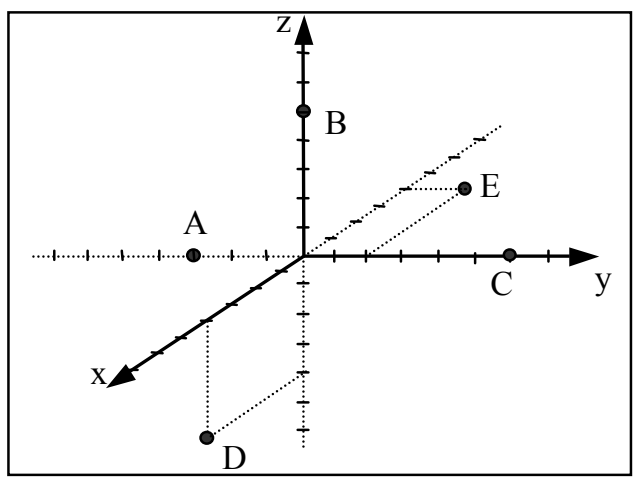

FIGURE 2. Diagram for post-test \#1. Students were asked to write $\bar{r}$ in terms of $\hat{r}, \hat{\theta}, \hat{\phi}$. The diagram in 2011 had just 3 points - A, B, \& C. Points D \& E were added subsequently.

S2: "I don't get it, how you can look at that and say $r$-bar is equal to one r-hat and know which direction it is... you have to have theta and phi to know exactly where r-bar is... So shouldn't that be included in the $r$ bar vector?"

They seem very troubled that the exact same symbol $\hat{r}$ is used to represent all three points from Fig. 1 . They know $\bar{r}$ points in different directions for those three points and necessarily has different $\theta$ and $\phi$, yet those angles appear nowhere in the final correct representation of just $5 \hat{r}$. Some wonder how you tell which way it's pointing by looking at just the symbol. Others even suggest that the symbol would be better written as explicitly including the angles: $\hat{r}(\theta, \phi)$.

A second major objection they raise is that if all the answers are the same, what is the point of it all?

S3: "So why do we even have all these conversions? Why do we have unit vectors? Why do we need to know which direction they point?"

S4: "What's the point of finding theta-hat and phihat if we don't even use them?"

This raises another implication for teaching and future research - we need to be ready to help students make sense of these ideas. Or perhaps we could head off the second objection before it even occurs by first practicing representing different $\bar{E}$ and $\bar{B}$ vector fields that have spherical and cylindrical symmetry with $\hat{\theta}$ and $\hat{\phi}$ before discussing $\bar{r}$. An example is the $B$ field of a very long wire that lies along the $z$-axis and carries a steady current $I$. They can work out that $\bar{B}=B_{o} \hat{\phi}$ represents the field for a given radius. But it's not clear that addressing their second objection will have any mitigating effects on their first. 


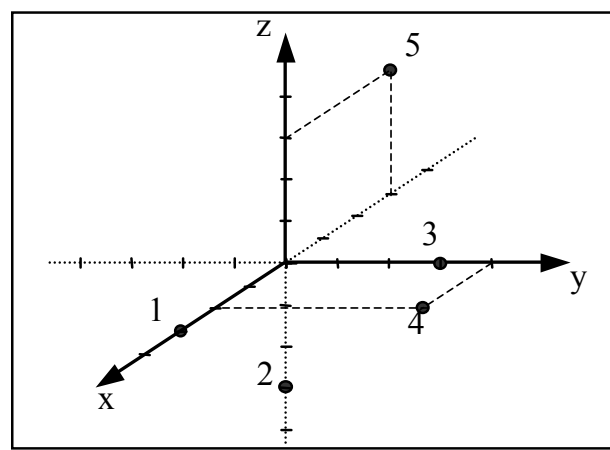

FIGURE 3. Diagram for post-test \#2. Students were asked to write $\bar{r}$ in terms of $\hat{s}, \hat{\phi}, \hat{z}$.

\section{POST-TESTS}

We gave two different post-tests to assess the effectiveness of the in-class group work. In 2011 and 2014, a month after the in-class work, students took an exam that had both post-tests on it. In 2017, two weeks after the in-class work, students took a quiz that had just the post-tests on it. Post-test \#1 (Fig. 2) assessed near transfer by asking students to again write the position vector in terms of $\hat{r}, \hat{\theta}, \hat{\phi}$. Note that points $\mathrm{A}, \mathrm{B}$, and $\mathrm{C}$ appeared on the pre-test, but were never discussed with the class afterwards. Post-test \#2 (Fig. 3 ) assessed for further transfer by asking students to write the position vector in terms of $\hat{s}, \hat{\phi}, \hat{z}$, a very different context. Tables 3 and 4 show the results for all three classes.

As reported in [2], the most common incorrect answer to the pre-test was of the form:

$$
\bar{r}=a \hat{r}+b \hat{\theta}+c \hat{\phi} \quad a, b, c \in \Re
$$

About half of all student answers had that form. The "Ln" columns in Tables 3 and 4 show the percent of students using that form on the post-tests. It is encouraging to see that for $\hat{r}, \hat{\theta}, \hat{\phi}$ (Table 3 ), the use of that form was reduced by at least half (to $25 \%$ ). Because of small $\mathrm{N}$, it is probably not possible to discern any reliable year-to-year patterns. But on average about $72 \%$ of students are using correct reasoning while only $11-15 \%$ of those who are not are using Eqn. (1). For $\hat{s}, \hat{\phi}, \hat{z}$ (Table 4), the use of Eqn. (1) is also cut by at

TABLE 3. Results from Post-test \#1 (Fig. 2) on spherical unit vectors

\begin{tabular}{c|rrr|rrr|rr|rrr|rrr|rrr|rr}
\hline & \multicolumn{3}{|c|}{ Point $\mathrm{A}^{3}$} & \multicolumn{4}{|c|}{ Point B } & \multicolumn{3}{|c|}{ Point C } & \multicolumn{3}{|c}{ Point D } & \multicolumn{3}{|c}{ Point E } \\
Year & Cr & Rs & Ln & Cr & Rs & Ln & Cr & Rs & Ln & Cr & Rs & Ln & Cr & Rs & Ln \\
\hline 2011 & 75 & 75 & 25 & 75 & 75 & 25 & 75 & 75 & 25 & - & - & - & - & - & - \\
2014 & 50 & 64 & 14 & 50 & 64 & 14 & 50 & 64 & 14 & 50 & 64 & 14 & 50 & 64 & 14 \\
2017 & 100 & 100 & 0 & 100 & 100 & 0 & 100 & 100 & 0 & 100 & 100 & 0 & 100 & 100 & 0 \\
\hline Totals & 65 & 73 & 15 & 65 & 73 & 15 & 65 & 73 & 15 & 61 & 72 & 11 & 61 & 72 & 11
\end{tabular}

${ }^{3} \mathrm{Cr}=\%$ of students who answered correctly with correct reasoning $\mathrm{Rs}=\%$ with correct reasoning (but could have math or value errors) $\mathrm{Ln}=\%$ who used the incorrect linear combination approach (see text)
TABLE 4. Results from Post-test \#2 (Fig. 3) on cylindrical unit vectors. (Table headings have the same meaning as for Table 3 ).

\begin{tabular}{r|rrr|rrr|rrr|rrr|rrr|rrr}
\hline & \multicolumn{3}{|c|}{ Point 1 } & \multicolumn{4}{|c|}{ Point 2 } & \multicolumn{4}{|c|}{ Point 3 } & \multicolumn{3}{c|}{ Point 4 } & \multicolumn{3}{c}{ Point 5 } \\
Year & Cr & Rs & Ln & Cr & Rs & Ln & Cr & Rs & Ln & Cr & Rs & Ln & Cr & Rs & Ln \\
\hline 2011 & 38 & 50 & 25 & 63 & 63 & 25 & 50 & 50 & 25 & 38 & 50 & 25 & 38 & 50 & 25 \\
2014 & 43 & 71 & 7 & 43 & 79 & 0 & 29 & 43 & 36 & 21 & 43 & 36 & 36 & 64 & 14 \\
2017 & 100 & 100 & 0 & 100 & 100 & 0 & 75 & 75 & 0 & 75 & 75 & 0 & 75 & 75 & 0 \\
\hline Totals & 50 & 69 & 12 & 58 & 77 & 8 & 38 & 50 & 31 & 38 & 50 & 31 & 42 & 62 & 19
\end{tabular}

least half for all but Points 3 and 4. For some reason, students in 2014 struggled with Point 3, even though it seems analogous to Point 1 . It is encouraging that even in the rather new context of $\hat{s}, \hat{\phi}, \hat{z}$, on average at least half of students used correct reasoning, and less than a third resorted to using the form of Eqn. (1).

\section{CONCLUSIONS}

In the future it would be good to have homework after the in-class work so that students can practice what they learned before taking a summative assessment. The homework for $\hat{r}, \hat{\theta}, \hat{\phi}$ could be to redo points 4, 5, and 6 from the pre-test. Post-test \#1 would then have to be suitably modified.

Only one student spontaneously drew triads of $\hat{r}, \hat{\theta}, \hat{\phi}$ or $\hat{s}, \hat{\phi}, \hat{z}$ in the post-tests to try to help them think about their final answers. We need to do more to help students use that representation, by adding it to homework and explicitly putting it on the post-test. Coordinating multiple representations is an excellent way to help students develop more robust knowledge networks and functional understanding.

Based on the results in Tables 3 and 4, it appears problems \#1 and \#2 done in a UA-MDM classroom have been effective in helping students gain some understanding of non-Cartesian unit vectors in the face of their initial strong negative reactions. But there is clearly still more work to do.

\section{ACKNOWLEDGMENTS}

Miki, Eads, \& Fenn; Michael Loverude for an invite; Steve Kanim \& Andrienne Traxler for mercy \& grace; Christos D, Jared D, Andy J, Beth T, referees \& God.

\section{REFERENCES}

1. D. J. Griffiths, Introduction to Electrodynamics, $1^{\text {st }}$ Edition, Upper Saddle River, N.J. : Prentice Hall, 1999.

2. B. E. Hinrichs, PERC 2010 Proceedings, 173-6.

3. B. E. Hinrichs, PERC 2013 Proceedings, 181-184.

4. E. Brewe, Am. J. Phys. 76 (12), 1155-1160 (2008).

5. D. M. Desbien, D.M., Ph.D. Thesis, ASU (2002).

6. E. Brewe, Phys.Rev.ST Phys.Educ.Res.7, 020106 (2011).

7. F. Reif and J. I. Heller, Educ. Psychol. 17, 102-27 (1982).

8. J. Durden, talk, AAPT Winter Meeting (2011). 